\title{
Pulmonary Embolism in Focus
}

\section{Rüdiger E. Scharf}

Division of Experimental and Clinical Hemostasis, Hemotherapy and Transfusion Medicine, and Hemophilia Comprehensive Care Center, Institute of Transplantation Diagnostics and Cell Therapy, Heinrich Heine University Medical Center, Düsseldorf, Germany
Venous thromboembolism (VTE), comprising deep-venous thrombosis (DVT) and pulmonary embolism $(\mathrm{PE})$, is a major healthcare burden worldwide. Specifically, VTE is associated with substantial morbidity and mortality. Despite improved thromboprophylaxis, the incidence of VTE has almost been constant over the past three decades (1).

\section{Epidemiology of PE}

Until recently, detailed data of the annual number of VTE events were hard to obtain. This is due to a number of circumstances and facts: VTE is often asymptomatic ('clinically silent'), misdiagnosed, or unrecognized at death. Moreover, the decreasing number of routine postmortem examinations prevents from proper diagnosis.

Using an epidemiological model to estimate the number of community- and hospital-acquired incidents of non-fatal VTE cases and VTE-related deaths, Cohen and colleagues of the VTE Impact Assessment Group in Europe (VITAE) (2) reported in 2007 that the total number of symptomatic VTE events per annum within six EU countries was

- 465,715 cases of DVT

- 295,982 cases of PE, and

- 370,012 VTE-related deaths.

Of these deaths, 126,145 (34\%) were due to sudden fatal PE, and 217,394 (59\%) followed undiagnosed PE, while an estimated

Correspondence to:

Univ.-Prof. Dr. Rüdiger E. Scharf, F.A.H.A.

E-mail: rscharf@uni-duesseldorf.de

Hämostaseologie 2018; 38: 7-8

received and accepted: January 23,2018
$27,473(7 \%)$ only were diagnosed ante mortem (2).

Basically, Heit and associates from the Mayo Clinic reported similar data for the United States throughout $(1,3,4)$. Accordingly, the incidence of VTE exceeds 1 per 1000; over 200,000 new cases of VTE occur in the US annually. Of these, about $30 \%$ die within 30 days; one-fifth suffer sudden death due to PE3. More recently, these numbers were reassessed.

The estimated annual incidence rates of VTE among individuals of European ancestry range from 104 to 183 per 100,000 person-years; incidence rates for PE (with or without DVT) range from 29 to 78 per 100,000 person-years; and corresponding rates for DVT alone (without $\mathrm{PE}$ ) range from 45 to 117 per 100,000 person-years $(1,4)$.

Other investigators in Western Europe, North America, Australia, and Southern Latin America have shown that the annual incidence rates of VTE range between 75 and 269 cases per 100,000 persons (for review [5]). In subjects 70 years of age or older, the VTE incidence rate can increase up to 700 per 100,000 persons (5).

\section{Diagnosis and management of acute PE}

Overall, the reported numbers and estimates clearly demonstrate the extent of the above-mentioned global 'healthcare burden' and also illustrate the challenge that we are facing, in particular when managing acute PE. Consequently, the ESC Guidelines on the diagnosis and management of acute pulmonary embolism were updated in 2014 by a Task Force of the European Society of Cardiology (ESC) under the leadership of Stavros V. Konstantinides $(6,7)$. More recently, he and his colleagues also reviewed advances in diagnosis, risk assessment, and treatment of acute PE and discussed current controversies $(5,8)$.

As outlined in the preceding editorial of this edition (9), Hämostaseologie - Progress in Haemostasis will reflect and reinforce some of the activities and advances in the management of $\mathrm{PE}$. The current issue therefore starts with two contributions from a series of reviews written by expert investigators upon invitation by Stavros V. Konstantinides, who also was in charge as Section Editor, managing the peer-review of the related manuscripts. I wish to express my gratitude to Stavros for organizing the update of PE in the Journal.

Marc Righini and Helia Robert-Ebadi present current algorithms that have improved diagnostic accuracy and made management of PE safer (10). The authors discuss the significance of clinical pretest prediction scores, D-dimer measurements and imaging tests, specifically the importance of computed tomography pulmonary angiography. Despite the advances in diagnostic procedures of VTE, some challenges persist in certain patient populations such as pregnant women.

Patients who have survived acute PE are facing an increased risk of chronic pulmonary dysfunction and a decreased quality of life. This so-called 'post-PE syndrome' includes chronic thromboembolic pulmonary hypertension (CTEPH). Frederikus A. Klok and Stefano Barco report that CTEPH can occur in up to $12 \%$ of patients after acute PE (11). The authors review pathological mechanisms and clinical conditions that are implicated in the transition of acute PE to CTEPH. Moreover, prediction, prevention and treatment of CTEPH and other manifestations of a 'post-PE syndrome' are discussed. The series update of PE will be continued in an upcoming issue of the Journal. Comments 
on the reviews by Letters to the Editor are most welcome.

In the current edition, Cristiana Bulato et al. report on a boy who suffered from unprovoked DVT and PE caused by an inherited protein C (PC) defect (12). His phenotype appeared consistent with a homozygous type IIA PC defect; however, genotyping of the propositus and family members revealed two distinct heterozygous gene lesions (i.e., missense mutations) corresponding to a compound PC defect. This report is a perfect example that careful characterization of the phenotype/genotype relationship will be required for proper classification of PC defects.

Zdeňka Hajšmanová et al. present a case report on their experience with idarucizumab to reverse the anticoagulant effect of dabigatran (13). The authors demonstrate that repeated administrations of the antidote are needed to overcome the direct inhibition of thrombin by dabigatran.

Misheng Zhao and co-workers identified a novel heterozygous $A \rightarrow C$ transition at nucleotide 5864 in the FGG gene of unrelated Chinese families, leading to a K232T substitution in the fibrinogen $\gamma$-chain (14). The investigators now report that expression of mutant $\gamma 232 \mathrm{~T}$ in trans- fected $\mathrm{CHO}$ cells is associated with a secretion defect of variant $\gamma 232 \mathrm{~T}$ fibrinogen (15). This observation may explain the reported hypofibrinogenemia in affected family members.

I wish to thank the authors for their contributions. I am also grateful to the Section Editors and referees involved in reviewing the manuscripts of this issue. The input by Stavros V. Konstantinides is specifically acknowledged.

\section{References}

1. Heit JA, Spencer FA, White RH. The epidemiology of venous thromboembolism. J Thromb Thrombolysis 2016; 41: 3-14.

2. Cohen AT, Agnelli G, Anderson FA, et al. Venous thromboembolism (VTE) in Europe. The number of VTE events and associated morbidity and mortality. Thromb Haemost 2007; 98: 756-764.

3. Heit JA, Silverstein MD, Mohr DN, et al. The epidemiology of venous thromboembolism in the community. Thromb Haemost 2001; 86: 452-463.

4. Heit JA. Epidemiology of venous thromboembolism. Nat Rev Cardiol 2015; 12: 464-474.

5. Konstantinides SV, Barco S, Lankeit M, Meyer G. Management of pulmonary embolism: an update. J Am Coll Cardiol 2016; 67: 976-990.

6. Konstantinides SV, Torbicki A, Agnelli G, et al. 2014 ESC guidelines on the diagnosis and management of acute pulmonary embolism. Eur Heart J 2014; 35: 3033-3069, 69a-69k.
7. Konstantinides SV. 2014 ESC Guidelines on the diagnosis and management of acute pulmonary embolism. Eur Heart J 2014; 35: 3145-3146.

8. Konstantinides SV. Trends in pulmonary embolism outcomes: are we really making progress? J Am Coll Cardiol 2016; 67: 171-173.

9. Scharf RE. Awareness and attraction or adhesion and activation. Hamostaseologie 2018; 38: 5-6.

10. Righini M, Robert-Ebadi H. Diagnosis of acute pulonary embolism. Hamostaseologie 2018; 38: 11-21.

11. Klok FA, Barco S. Follow-up after acute pulmonary embolism. Predicting chronic thromboembolic pulmonary hypertension and post-pulmonary embolism syndrome. Hamostaseologie 2018; 38: 22-32.

12. Bulato C, Campello E, Gavasso S, Maggiolo S, Tormene D, Simioni P. Peculiar laboratory phenotype/ genotype relationship due to compound inherited protein $\mathrm{C}$ defects in a child with severe venous thromboembolism. Hamostaseologie 2018; 38: 33-38.

13. Hajsmanová Z, Sigutová $\mathrm{P}$, Lavicková $\mathrm{A}$. Repeated administration of idarucizumab to a patient with dabigatran overdose. Hamostaseologie 2018; 38: 39-42.

14. Zhu L, Wang Y, Zhao M, et al. Novel mutations (gammaTrp208Leu and gammaLys232Thr) leading to congenital hypofibrinogenemia in two unrelated Chinese families. Blood Coagul Fibrinolysis 2014; 25: 894-897.

15. Zhao M, Wang M, Lou Z, et al. Congenital hypofibrinogenemia associated with gamma-K232T. In vitro expression demonstrates defective secretion of the variant fibrinogen. Hamostaseologie 2018; 38: 43-48. 